\title{
Manipulation of the Longitudinal Profile
}

\author{
R. Tikhoplav*, G. Kazakevich ${ }^{\dagger}$, D. Mehalcea** and P. Piot** \\ *Department of Physics and Astronomy, University of California, Los Angeles, CA 90095 \\ ${ }^{\dagger}$ Fermi National Accelerator Laboratory, Batavia, IL 60510 \\ ${ }^{* *}$ Northern Illinois University Department of Physics, DeKalb, IL 60115
}

\begin{abstract}
We present studies of transverse emittances and electron beam pulse length for various operating points of the electron source: electron beam charge, laser length and spot size, and solenoid settings. We especially study the impact, on transverse emittance, of short Gaussian versus long square temporal distributions of the photocathode drive-laser.
\end{abstract}

Keywords: linear accelerator, beam emittance, photoinjector, pulse stacker

PACS: $29.27-\mathrm{a}, 41.75 . \mathrm{Ht}, 42.60 . J f$

Optimization of the UV laser pulse shape on the cathode should be relevant in reducing the space charge effects of the electron beam in a photoinjector linac. The simplest way to reduce the charge density is to increase the laser spot size, but this results in increased emittance. A more effective way would be to elongate the laser pulse, while also making its longitudinal profile square. One of the most efficient shapes for reducing the space charge effect is a "can" shape ${ }^{1}$, which has a square profile in both the transverse and longitudinal domains. In the transverse domain a quasi-square shape is relatively easy to achieve by introducing an iris in the laser beam and imaging the iris onto the cathode. However, in the longitudinal domain this is not such an easy task. To generate a long and flat-topped temporal distribution we use a "pulse stacker". In this device the photoinjector laser beam is split into four different beams which are then recombined (stacked) after a time delay is introduced between the pulses, this results in a quasi flat-top pulse.

\section{PULSE STACKER}

We use a modified pulse stacker (see Fig. 1) analogous to the one used at DESY [1]. A Gaussian laser pulse is split in two by a prism $(\mathrm{Pz})$ so that the P-polarized beam goes straight and the $\mathrm{S}$-polarized beam is reflected sideways. Each of the beams (P and $\mathrm{S}$ ) hit the set of mirrors $(\mathrm{M})$ and then they are recombined by another prism $(\mathrm{Pz})$; one set of mirrors is positioned on a movable platform so that the delay between the two beams can be adjusted. After the first stage of splitting and recombining the double Gaussian goes through a second stage analogous to the one described above. Half-wave plates $(\lambda / 2)$ are used to adjust the relative intensities of the stacked Gaussians; intensity adjustments

\footnotetext{
1 An ellipsoid of revolution is the best possible shape since, ideally it provides no emittance growth due to the space charge effect. A laser pulse of such shape, however, is very challenging to obtain.
} 


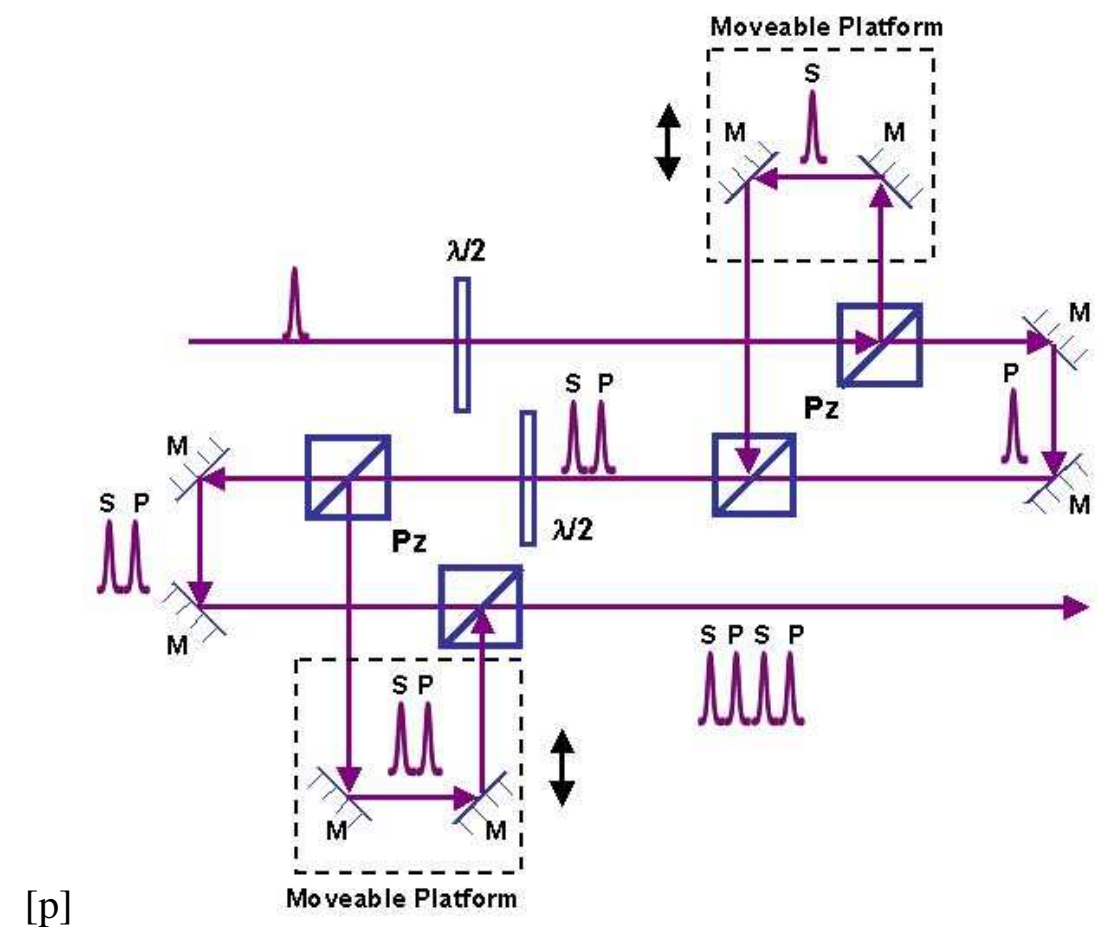

FIGURE 1. Pulse Stacker; $M$-mirror, $\lambda / 2$-halfwave plate, Pz-prism, SP-alternating polarizations.

along with the adjustable delay enable us to optimize the flat-top shape. Each stacking stage makes two copies of the input and then stacks them; so, in the most general case when we have $n$ stacking stages the final number of copies utilized is $2^{n}$.

One of the features of this design is that the copies utilized by each stacking stage have different polarization. Thus we are able to avoid the longitudinal interference between adjacent pulses by use of the alternate polarization scheme: SPSP. Another feature is that all the input intensity appears in the stacked pulse (in older designs half of the light was lost [2]).

The pulse stacker has been successfully built and commissioned at the Fermilab NICADD Photoinjector Laboratory (FNPL). The use of the imaging system reduces the transverse fluctuation of the stacked beam spot centroids with the respect to each other, when they reach the cathode. The resulting quasi flat-top pulse length is 20.8 ps full width half max (FWHM) which as expected, is four times longer than the original single Gaussian (see Fig. 2). The overall transmission through the pulse stacker is about $80 \%$, the intensity loss is due to the fact that the laser beam encounters sixteen surfaces while passing through the pulse stacker. The loss could be slightly reduced by substituting Brewster's plates for the prisms.

The stacked pulse "flatness" (the variation of the intensity in the flat-top region) is better than $5 \%$ rms. The rise time $(10 \%-90 \%)$ is $6.4 \mathrm{ps}$; of course, the rise-time is completely determined by the original Gaussian pulse shape. In order to make the pulse more square, that is to decrease the ratio of the rise-time over FWHM, we have been considering adding one more stacking stage, thus increasing the FWHM by a factor of two. The flat-top pulse length depends on the number of pulses stacked and on the delay 

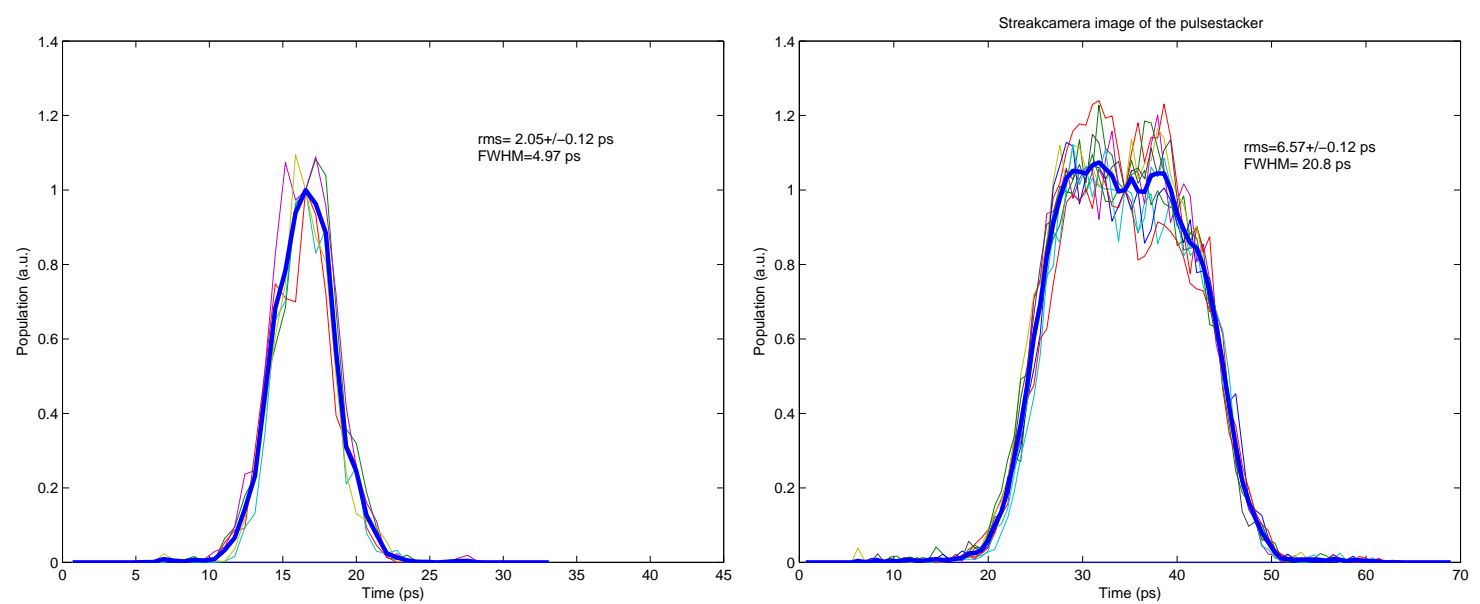

FIGURE 2. Time profile of the UV laser pulse measured by a streak camera. Five Gaussian (4.97 ps FWHM, left) and eight flat-topped (20.8 ps FWHM, right) laser shots and their average (thick line).

between them; the overall pulse length can be varied from 8 to 25 ps FWHM, by stacking two or four pulses. It is not advisable to have more than three stacking stages since the pulse length must be much smaller than the rf period, which is $769 \mathrm{ps}$; otherwise the energy spread increases resulting in increased longitudinal emittance (the longitudinal emittance is proportional to the cube of the pulse length [3]).

\section{EMITTANCE MEASUREMENTS}

In the most general case, different parts of an electron bunch have different values of emittance. Usually, an integrated emittance (referred to as "projected" emittance) is considered as a comparative characteristic of a beam; integration is performed in both the transverse and time domains. One can also consider measurements of the slice emittance, resolved in the time domain but integrated over the transverse dimentions (see [4], [5]). The transverse emittance distribution as well as the dependence of the integrated emittance on different parameters (such as charge and shape of the beam) is being considered in the following sections.

\section{Experimental Set-up}

The emittance was measured by using the slit technique. Slits, 50 micron wide, were made in an tungsten mask; the slit separation was $1 \mathrm{~mm}$. The mask is remotely interchangeable with an optical transition radiation (OTR) Al screen (which allows us to observe the transverse bunch size by means of a CCD camera) and located at flag $\mathrm{X} 3$ of the FNPL beam line. The image of the slits can be viewed on an OTR yttrium aluminum garnet (YAG) screen located at flag X5. The distance between the flags is $78 \mathrm{~cm}$. The beam divergence is calculated from the growth of the size of the slit image for a given flag distance. Beam spot size, its divergence as well as its energy determine 


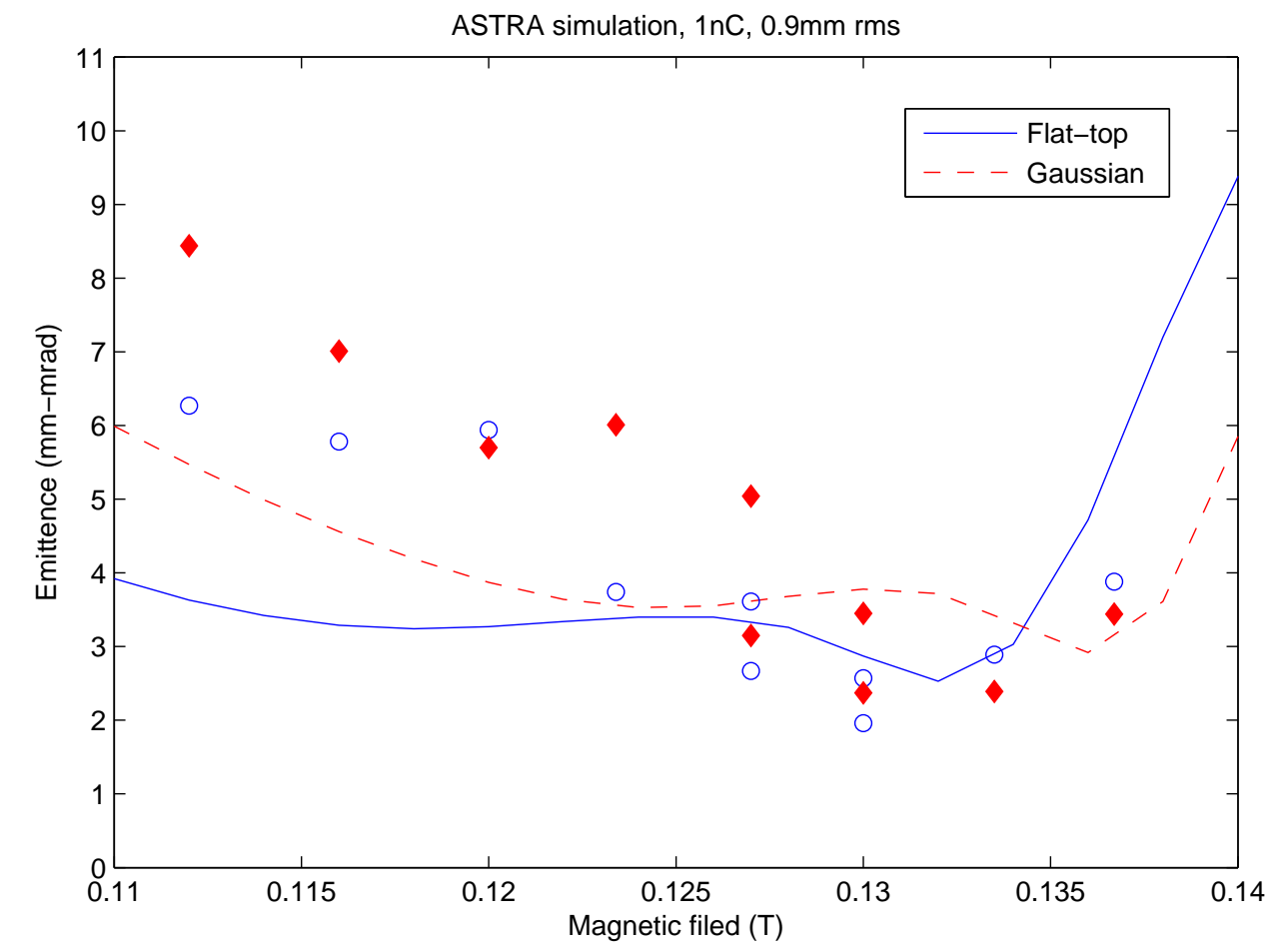

FIGURE 3. Emittance versus solenoid field: experimental points for Gaussian (diamonds) and flat-top (circles), and ASTRA simulation (dashed and solid lines respectively). Gaussian pulse length is 3.5 ps rms and flat-top is $6.5 \mathrm{ps} \mathrm{rms}$; the transverse size for both is $0.9 \mathrm{~mm} \mathrm{rms}$ and the charge is $1 \mathrm{nC}$.

the normalized emittance:

$$
\varepsilon_{x}=\beta \gamma \sigma_{x} \sigma_{x^{\prime}}
$$

where $\sigma_{x}$ is the rms beam size (flag X3) and $\sigma_{x^{\prime}}=d_{x} / L$ is the rms value of the beam divergence; $d_{x}$ is the rms value of the slit image width (flag X5) and $L$ is the distance between the flags. The FNPL linac was set to operate at its regular regime; see Table 1 for the list of parameters.

The emittance compensation scheme proposed by Carlsten [6] was used to minimize the projected emittance. There are three solenoids around the FNPL rf-gun: primary, bucking and secondary. The emittance measurements were taken while varying the solenoid current in such a way that all three solenoids had the same current. This ensures that the magnetic field on the photocathode surface is zero. The current was varied from 210 to 280 Amps (which corresponds to peak fields from 0.112 to 0.137 Tesla).

\section{Results}

We have measured the emittance for both single (Gaussian) and stacked (flat-top) pulses as a function of rf-gun solenoid current (see Fig. 3). The experimental results were compared with ASTRA simulation [7], which tracks particles through user defined 
TABLE 1. FNPL operating parameters.

\begin{tabular}{|l|l|}
\hline Laser transverse size on photocathode, $\mathrm{rms}$ & $0.91 \mathrm{~mm}$ \\
\hline Laser pulse length (single Gaussian), rms & $2.05 \mathrm{ps}$ \\
\hline Laser pulse length (flat-top), rms & $6.57 \mathrm{ps}$ \\
\hline Charge & $1.013 \mathrm{nC}$ \\
\hline rf-gun peak field & $34.82 \mathrm{MV} / \mathrm{m}$ \\
\hline 9-cell accel. field & $12.00 \mathrm{MV} / \mathrm{m}$ \\
\hline Electron energy & $15.83 \mathrm{MeV}$ \\
\hline Separation of pulses in train & $1 \mu \mathrm{s}$ \\
\hline Number of pulses in train & 2 \\
\hline Repetition rate & $1 \mathrm{~Hz}$ \\
\hline rf frequency & $1.30 \mathrm{GHz}$ \\
\hline Laser injection phase & $45^{\circ} \pm 5^{\circ}$ \\
\hline 9-cell phase & $\sim 84^{\circ}$ \\
\hline
\end{tabular}

external fields taking into account the space charge field of the particle cloud. The graphs were plotted using Matlab [8]. The minimum value for a Gaussian pulse is $2.9 \mathrm{~mm}$ $\operatorname{mrad}(0.137 \mathrm{~T})$; and for a stacked pulse it is $2.5 \mathrm{~mm}-\mathrm{mrad}(0.132 \mathrm{~T})$. The stacked pulse has $\sim 20 \%$ lower emittance and agrees well with the ASTRA simulation. In a properly optimized accelerator, that is in accelerator which is not only tuned properly, but also uses optimized hardware (including the proper distance between the rf-gun and the 9cell cavity), we expect a sub $1 \mathrm{~mm}$-mrad emittance (for $1 \mathrm{nC}$ charge). For such a low emittance machine, utilization of a flat-top scheme would be a great improvement. Also, for such a machine, we expect a better performance for the flat-top scheme in a super large charge regime (greater than $10 \mathrm{nC}$ ).

Phase space correlation (divergence as a function of transverse distance from the beam centroid) has been analyzed for one of the settings (flat-top, $0.137 \mathrm{~T}$ ) see Fig. 4. As expected the divergence is smallest in the center of the beam and it grows as we move away from the center (transversely).

\section{PULSE LENGTH AS A FUNCTION OF CHARGE}

Even though the set charge for the International Linear Collider was chosen to be $3.2 \mathrm{nC}$, there are experiments that require drastically different values of charge. Experiments sensitive to emittance would typically require a charge less than $1 \mathrm{nC}$. On the other hand, there are number of experiments (such as acceleration by a plasma wakefield) that require very large charge (greater than $10 \mathrm{nC}$ ). It is understood that the transverse emittance grows with charge due to the space-charge effect. However, it is also interesting to observe how the space-charge contributes to the pulse elongation and whether such elongation depends on the original shape of the pulse.

The laser intensity was varied by means of a half-wave plate and a prism. Thus we 

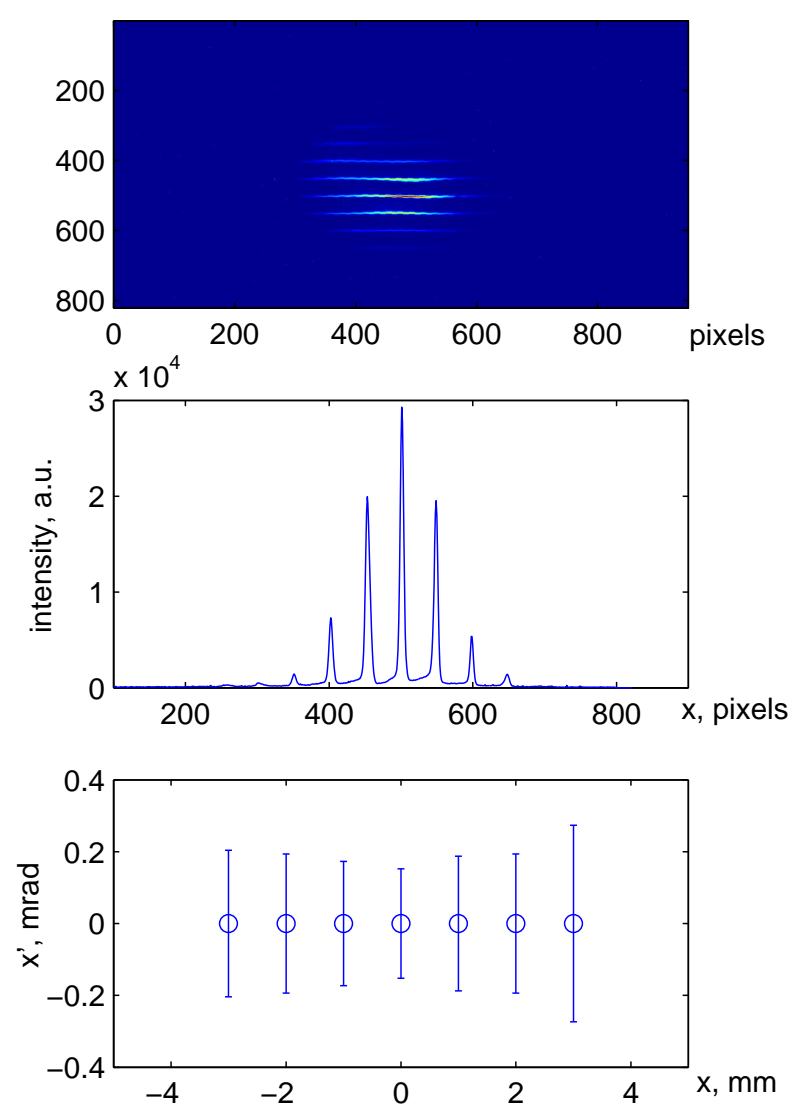

FIGURE 4. Image of slits at flag X5 (top) and its projection on the vertical axis (middle). Phase space correlation: divergence as a function of transverse distance (bottom).

were able to vary the charge of the electron bunch from $1 \mathrm{nC}$ to $10 \mathrm{nC}$. The aperture was set to $1.77 \mathrm{~mm}$ rms to better accommodate such a range of charges and then imaged onto the photo cathode so that diffraction effects due to the aperture were avoided. The streak camera was used to collect the light from the OTR. The dependence of pulse length on bunch charge was measured for both Gaussian and stacked pulses and is presented on Fig. 5. The pulse length for the Gaussian distribution has a stronger charge dependence. It agrees well with ASTRA simulation. This is due to the stronger space charge effect for the Gaussian distribution.

\section{CONCLUSION}

A long laser pulse with a quasi flat-top temporal distribution was constructed by adding four single Gaussians by means of a pulse stacker. Such a laser pulse (as well as a single Gaussian) was imaged onto the photocathode and an electron pulse with corresponding temporal distribution was produced. The two temporal distributions have been compared analytically and experimentally. The minimum emittance was found for each of them by scanning the rf-gun solenoid current. The quasi flat-top emittance gives $20 \%$ better 


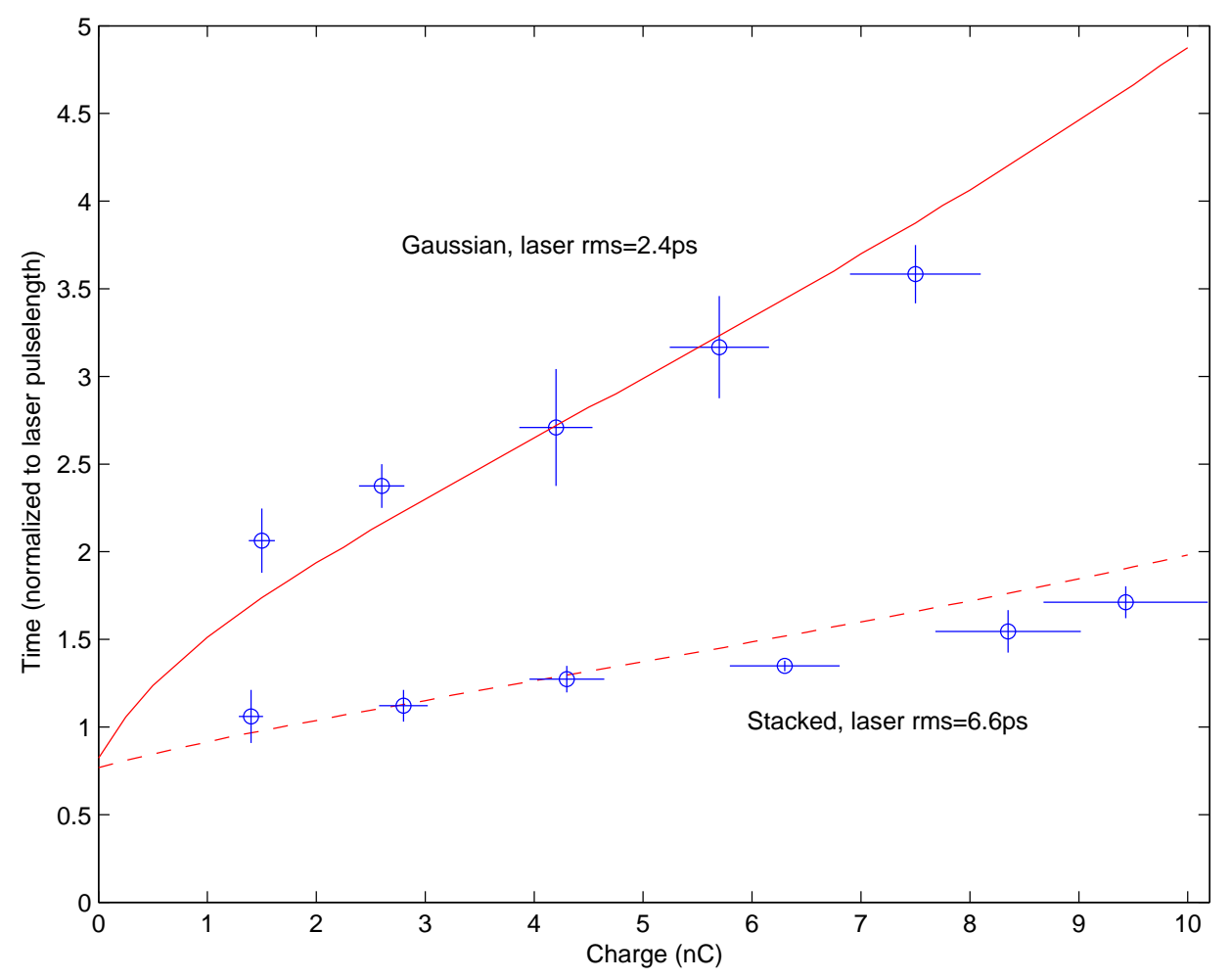

FIGURE 5. Electron bunch length normalized to the corresponding laser pulse length (2.4 ps rms for Gaussian and $6.6 \mathrm{ps}$ rms for the flat-top) as a function of charge. The growth for the longer flat-top pulse is significantly slower.

value. Electron pulse elongation as a function of bunch charge was studied for both distributions. The growth for the longer flat-top pulse is significantly slower.

A quasi flat-top distribution in the time domain yields better results than a simple Gaussian for both the emittance measurements and space-charge effects. It is therefore preferable to use the pulse stacker for future experiments especially those requiring low emittance and/or high charge.

\section{REFERENCES}

1. S. Schreiber, TESLA Collaboration Meeting, WG3 (2004).

2. M.J. Fitch, Ph.D Dissertation, UR-1628, 2000

3. K.-J. Kim, Nucl. Instr. Meth. A 275, 201-218 (1989).

4. X. Qiu et al., Phys. Rev. Lett. 76, p. 3723 (1996).

5. S. Giermann, Ph.D Dissertation, UC San Diego (2001).

6. B.E. Carlsten, Nucl. Instr. Meth. A 285, p. 313 (1989).

7. K. Flöttmann, ASTRA user manual, DESY (2000). (information available on the web site http://www. desy. de/ $\sim \operatorname{mpy} f l o$ )

8. Matlab is available from MathWorks, Inc. 